\title{
CICATRIZAÇÃO DE FERIDAS: ESTUDO COMPARATIVO EM RATOS HIPERTENSOS NÃO TRATADOS E TRATADOS COM INIBIDOR DA ENZIMA CONVERSORA DA ANGIOTENSINA
}

\author{
WOUND HEALING: COMPARATIVE STUDY IN HYPERTENSIVE RATS UNTREATED \\ AND TREATED WITH AN ANGIOTENSIN CONVERTING ENZIME INHIBITOR
}

\author{
Maria de Lourdes Pessole Biondo-Simões ${ }^{1}$; Emanuelle de Mello Alcantara ${ }^{2}$; \\ Juliana Corrêa Dallagnol ${ }^{3}$; Kelly Okamoto Yoshizumi ${ }^{3}$; \\ Luiz Fernando Bleggi Torres ${ }^{4}$; Karin Soldatelli Borsato ${ }^{5}$
}

\begin{abstract}
RESUMO: Objetivo: Reconhecer a interferência do captopril na cicatrização de feridas cutâneas de ratos hipertensos. Métodos: Distribuíram-se 111 ratos em quatro grupos: controle normotenso $(\mathrm{N}=30)$; controle hipertenso $(\mathrm{N}=30)$, os quais receberam $1 \mathrm{ml} / \mathrm{dia}$ de solução de cloreto de sódio a $0.9 \%$ por via oral; grupo experimento $(\mathrm{N}=31)$, hipertensos que receberam $7,5 \mathrm{mg} / \mathrm{kg} / \mathrm{dia}$ de captopril e um grupo aferição $(\mathrm{N}=20), 10$ hipertensos e 10 normotensos, nos quais aferiu-se a pressão na aorta abdominal, no último dia de experimento. Após 15 dias de medicação, fez-se uma incisão da pele e da tela subcutânea, na região médio-dorsal dos grupos I, II e III, seguida de síntese. Ressecaram-se as cicatrizes de 10 animais de cada grupo, no $4 .^{\circ}, 7 .^{\circ}$ e $14 .^{\circ}$ dias após a operação, que divididas em duas partes foram enviadas para a tensiometria e para análise histológica. Resultados: A pressão arterial média de 83,18 $\pm 7,51$ mmHg nos normotensos e $151,36 \pm 10,51 \mathrm{mmHg}$ nos hipertensos. As cicatrizes dos hipertensos tratados e não tratados eram menos resistentes que as dos normotensos, nos tempos iniciais $(\mathrm{p}<0,05)$ e que ao $14 .^{\circ}$ dia as resistências se igualaram. Não houve diferença entre o grupo tratado e o não tratado. A densidade de colágeno total foi maior nos normotensos em todos os tempos $(\mathrm{p}<0,05)$ e não houve diferença entre hipertensos tratados e não tratados. A epitelização, a reação inflamatória e a formação do tecido de granulação foi semelhante nos três grupos. Conclusão: O captopril, em ratos, não modifica a cicatrização, ficando as diferenças relacionadas à hipertensão (Rev. Col. Bras. Cir. 2006; 33(2): 74-78).
\end{abstract}

Descritores: Cicatrização de feridas; Pele; Hipertensão; Captopril; Colágeno.

\section{INTRODUÇÃO}

Considerando-se que o processo de cicatrização é um fenômeno local do qual participam elementos comuns a vários setores do organismo, é fácil imaginar que fatores ambientais e fisiológicos exerçam grande impacto na evolução da cicatrização, podendo exercer influência na qualidade da cicatriz, no tempo de cicatrização e na presença ou não de complicações.

Sabendo disto, questionou-se se as drogas utilizadas no tratamento de co-morbidades sistêmicas como a hipertensão arterial sistêmica (HAS) poderiam ou não interferir no processo de cicatrização. Para tanto, optou-se por estudar a interferência do captopril, droga amplamente utilizada no tratamento da HAS, no processo de cicatrização de feridas de pele de ratos.

A hipertensão arterial é doença de alta prevalência mundial.É, provavelmente, o problema de saúde pública mais importante nos países desenvolvidos ${ }^{1}$.
A hipertensão renovascular é a forma secundária mais comum de hipertensão e não é facilmente reconhecida. Uma redução da pressão de perfusão renal em $50 \%$ conduz ao aumento imediato e persistente da secreção de renina decorrente da isquemia renal, com supressão da secreção contralateral ${ }^{2}$.

O objetivo do tratamento da hipertensão é reduzir o risco de morbidade e de mortalidade devida às lesões em órgãos-alvo que este distúrbio pode levar. O uso de inibidores da enzima conversora de angiotensina (IECA) é uma importante opção terapêutica. Na hipertensão renovascular causada por estenose unilateral, os IECA são úteis, atuando na inibição da formação de angiotensina II e bloqueando o sistema renina-angiotensina.

O captopril foi o primeiro IECA descoberto e ainda é considerado o medicamento padrão ouro nesta classe de drogas anti-hipertensivas².

Sabe-se que portadores de HAS apresentam alterações vasculares na tela subcutânea e da arquitetura da matriz extra-celular. A atividade inflamatória e a síntese tecidual são

\footnotetext{
1. Doutora em Cirurgia Experimental pela UNIFESP-EPM; Professora Titular da Disciplina de Metodologia da Pesquisa da PUCPR.

2. Farmacêutica pela Universidade Tuiuti do Paraná e aluna do Programa de Iniciação Científica do Curso de Medicina da PUCPR.

3. Alunas do Programa de Iniciação Científica do Curso de Medicina da PUCPR.

4. Doctor of Philosophy (PhD), Department of Neuropathology, Institute of Neurology, The National Hospital for Nervous Diseases, Queen Square London, Inglaterra; Professor Titular da Disciplina de Anatomia Patológica da PUCPR.

5. Doutora em Engenharia e Ciência de Materiais pela UFSC; Professora Titular do Curso de Engenharia Mecânica da PUCPR.

Recebido em 14/10/2005

Aceito para publicação em 15/12/2005

Conflito de interesse: nenhum

Fonte de financiamento: Bolsa PIBIC-PUCPR

Trabalho realizado no Laboratório de Farmacologia da Pontifícia Universidade Católica do Paraná (PUCPR) e no Laboratório de Patologia Experimental da PUCPR.
} 
oxigênio-dependentes e desta forma pacientes com HAS poderiam ter o processo de reparação de feridas prejudicado.

Neste estudo busca-se reconhecer a interferência da hipertensão arterial não tratada e da hipertensão arterial tratada com inibidor da enzima conversora da angiotensina (captopril) no processo de cicatrização de feridas cutâneas, em ratos.

\section{MÉTODO}

Para a realização deste estudo obedeceu-se à Lei Federal 6.638 e as orientações do Colégio Brasileiro de Experimentação Animal. O projeto desta pesquisa foi aprovado pelo Comitê de Ética em Pesquisa com Animais da PUCPR em reunião realizada no dia 5 de outubro de 2004, atendendo aos aspectos das Resoluções em vigor sobre as Diretrizes e Normas Regulamentadoras de Pesquisa envolvendo animais e recebendo o número 005/03.

Utilizaram-se 111 ratos (Rattus norvegicus albinus. Rodentia mammalia), da linhagem Wistar, machos, adultos, provenientes do Biotério Central da PUCPR

Mantiveram-se os animais no Biotério Central da Pontifícia Universidade Católica do Paraná onde o macro-ambiente é semi-controlado, com foto-período de 12 horas, temperatura ambiental média de $21 \pm 2^{\circ} \mathrm{C}$ e a intensidade de ruído e a umidade relativa do ar são as do ambiente geral. Distribuíram-se, as gaiolas, todas à mesma distância da fonte de luz e os ratos receberam cuidados diários, água potável e ração padrão para a espécie à vontade.

Marcaram-se e pesaram-se os animais no primeiro dia do experimento para cálculo da dose do hipotensor.

Conseguiu-se a anestesia com injeção intra-muscular de 0,2 ml para cada 100 gramas de peso da mistura de um mililitro de quetamina $(50 \mathrm{mg} / \mathrm{ml})$ com um mililitro de xilazina $(20$ $\mathrm{mg} / \mathrm{ml}$ ), para todos os procedimentos dolorosos e/ou situações de estresse.

A amostra foi distribuída, aleatoriamente, em quatro grupos: grupo controle normotenso (30 ratos), submetidos à laparotomia simulada para os quais, 48 horas após a operação foi iniciada a administração de um mililitro de solução de cloreto de sódio à $0,9 \%$, por sonda oro-gástrica; grupo controle hipertenso (30 ratos), submetidos à laparotomia com indução da hipertensão arterial, fazendo-se a estenose da artéria renal esquerda e aos quais, após 48 horas, iniciou-se a administração de um mililitro de solução de cloreto de sódio à $0,9 \%$ por sonda oro-gástrica; grupo experimento (31 ratos): submetidos à operação para indução da hipertensão arterial e aos quais se iniciou, após 48 horas a administração do anti-hipertensivo captopril, 7,5 mg/Kg/dia; grupo aferição da pressão arterial (20 ratos): 10 com hipertensão renovascular provocada por estenose parcial induzida da artéria renal esquerda e 10 sem hipertensão . Todos os animais receberam um mililitro ao dia de solução salina de $\mathrm{NaCl}$ a $0,9 \%$ por via oral. No último dia de experimento foi aferida a pressão arterial média, na aorta abdominal, para comprovar a eficácia do método de indução de hipertensão.

Sob anestesia, induziu-se a hipertensão fazendo-se a estenose da artéria renal esquerda. Aguardou-se dois dias para o início da administração do anti-hipertensivo (captopril) e da solução salina isotônica de $\mathrm{NaCl}$ a $0,9 \%$ para os respectivos grupos.

Por um período de 15 dias os grupos receberam a solução estabelecida, sempre na mesma hora do dia e permaneceram recebendo-a até a data prevista para verificação dos resultados.

Ao final dos 15 dias foi feita uma incisão de $4 \mathrm{~cm}$ na pele do dorso de todos os animais, colocada na linha média longitudinal e que se iniciava logo abaixo da base da inserção das patas dianteiras. Promoveu-se a síntese aplicando-se 4 pontos simples de fio monofilamentar de nylon 4.0.

Para aferição submeteram-se à eutanásia, 10 animais de cada grupo no $4 .^{\circ}, 7^{\circ}$ e $14 .^{\circ}$ dia após a operação. Ressecava-se a cicatriz, com $0,5 \mathrm{~cm}$ das margens superior e inferior e 2 $\mathrm{cm}$ das margens laterais, distendendo o retalho sobre papel filtro. Desconsideraram-se os $0,5 \mathrm{~cm}$ superiores e inferiores da cicatriz. Dividiram-se as peças em duas partes iguais, uma delas para o ensaio de tração (tensiometria) e a outra parte, fixada em formalina tamponada, para a análise histológica. Processaram-se as peças e obtiveram-se cortes com quatro micrômetros de espessura que corados pela Hematoxilina e Eosina permitiram reconhecer os aspectos histológicos gerais e pelo Sirius-red a densidade do colágeno e a maturação da cicatriz.

Os testes de força máxima (medida em Newton) e de deslocamento (em milímetros) para o rompimento da cicatriz de pele foram realizados com a utilização de máquina de tração EMIC $^{\circledR}$ do Laboratório de Ensaios Destrutivos da PUC-PR, conectada a microcomputador. Considerou-se força máxima a maior força ou carga aplicada no corpo de prova até sua ruptura. Aplicaram-se as forças sempre perpendiculares à cicatriz, tentando afastar suas bordas.

Na microscopia óptica os cortes tratados pela coloração Sirius Red foram analisados, com 200 aumentos, utilizando-se microscópio óptico Olimpus ${ }^{\circledR}$ BX-50 com luz polarizada. Nesta coloração, as fibras colágenas mais espessas e fortemente birrefringentes representam o colágeno I, e se mostram coradas em tons de amarelo a vermelho e as fibras do colágeno III, mais finas e dispersas, fracamente birrefringentes apresentam-se coradas em tons de verde. A obtenção das imagens foram realizadas por câmera Sony CCDIRIS, sendo enviadas ao monitor Dell, congeladas e analisadas através do aplicativo Image Pró-Plus ${ }^{\circledR} 4.0$ para Windows em computador Intel Pentium 4. As percentagens ocupadas pelas fibras do tipo I e III foram analisadas e calculadas em três campos, sendo, então, obtida uma média da leitura daquele corte.

$\mathrm{Na}$ análise histológica avaliou-se a epitelização, a reação inflamatória, a existência de tecido de granulação e de fibrose. Utilizou-se para a análise os seguintes critérios:

- Epitelização: incompleta, quando existia crosta fibrinoleucocitária sobre a ferida com epitélio descontínuo, e completa quando não existia solução de continuidade epitelial;

- Reação Inflamatória: acentuada quando existia infiltração de células inflamatórias visível ao pequeno aumento do microscópio óptico $(10 \mathrm{X})$, moderada quando a identifica- 
ção do infiltrado neutrofílico exigia o aumento de $100 \mathrm{X}$ e discreta quando era preciso o aumento de $200 \mathrm{X}$.

- Tecido de Granulação: tipo A, caracterizado por grande quantidade de fibroblastos em pequena matriz colágena, sem edema, pouco visível com aumento de $10 \mathrm{X}$ e pequena quantidade de vasos, tipo B, tecido de granulação edemaciado, evidentemente visível com aumento de $10 \mathrm{X}$, grande quantidade de vasos, indicando neoangiogênese acentuada.

- Fibrose: quando poucos núcleos de fibroblastos estavam presentes em evidente matriz colágena organizada, com ausência de processo inflamatório e raros vasos capilares presentes, indicando avançado grau de cicatrização.

Avaliou-se, ainda a presença ou não de granuloma de corpo estranho que indicaria resíduo de fio ou pêlo do animal na cicatriz.

No $14 .^{\circ}$ dia aferiu-se a pressão arterial da aorta abdominal dos 20 animais do grupo aferição, 10 com estenose da artéria renal esquerda e 10 sem estenose.

Os resultados foram submetidos à tratamento estatístico utilizando-se para a análise de médias, entre os grupos, para resultados não paramétricos o teste de Mann Whitney e para os paramétricos o teste $\mathrm{t}$ de student; e para as tabelas $2 \mathrm{x}$ 2 o teste exato de Fisher. Fixou-se p $\leq 0,05$ ou 5,0\% como limite para rejeição da hipótese de nulidade.

\section{RESULTADOS}

Durante o período de estudo registrou-se o óbito de três animais do grupo controle hipertenso, isto é, hipertensos não tratados e um do grupo dos hipertensos tratados com captopril. Entre os mortos, do grupo controle, observou-se a presença de sangramento nasal, de hematúria e de hemorragia ocular, levando a supor que a causa tenha sido a hipertensão. O óbito do animal do grupo tratado ocorreu por acidente anestésico.

Observou-se que o grupo de controle normotenso apresentou cicatrizes mais resistentes nos dois tempos iniciais e, embora no $14 .^{\circ}$ ainda fossem mais resistentes, a diferença não era significante. Não se verificaram diferenças significantes de força máxima ou deslocamento entre os grupos hipertensos nos três tempos estudados. Na Figura 1 temse o gráfico das médias das forças máximas obtidas nos três grupos e nos três tempos.

A densidade do colágeno e das frações de colágeno I e III foram significativamente maiores no grupo controle normotenso. Não houve diferença entre controle hipertenso e hipertensos tratados com captopril. A Figura 2 ilustra as densidades de colágeno I, III e total, nos três tempos e nos três grupos estudados e a Figura 3 mostra aspectos histológicos do colágeno.

A epitelização, a intensidade da reação inflamatória e da formação de tecido de granulação foi semelhante nos dois grupos.

A avaliação do grupo aferição serviu para determinar os níveis pressóricos dos animais. Todos os submetidos à estenose da artéria renal desenvolveram hipertensão. Enquanto a pressão arterial média no controle era de 83,18 $\pm 7,51$ $\mathrm{mmHg}$, no experimento foi de $151,36 \pm 10,51 \mathrm{mmHg}$.

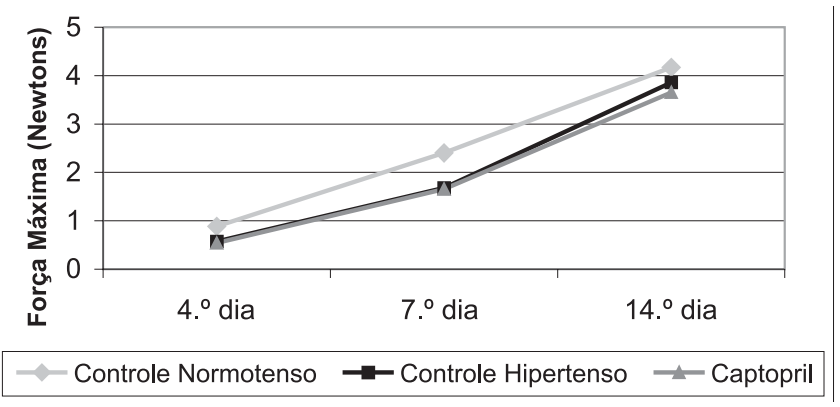

Figura 1 - Gráfico demonstrativo das médias das forças máximas de ruptura nos 3 tempos e nos 3 grupos.

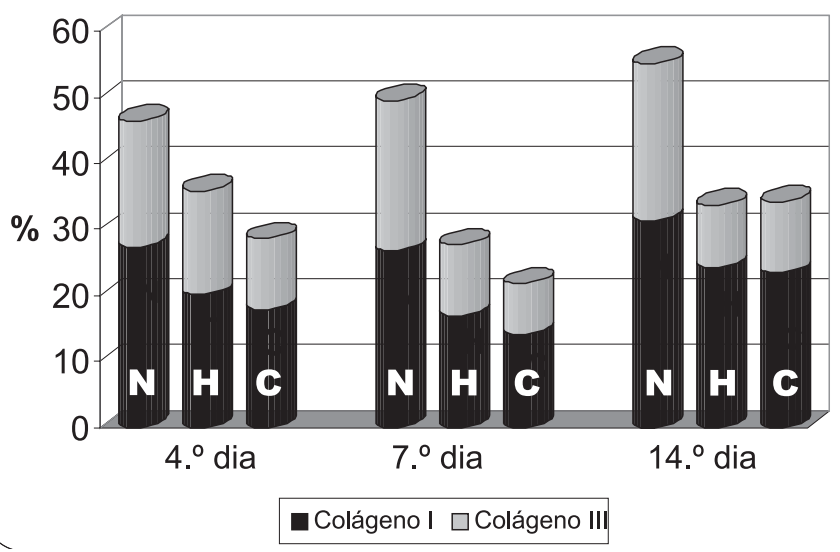

Figura 2 - Gráfico demonstrativo das densidades de colágeno I, III e total (somatória de I e III) nos 3 tempos e nos 3 grupos. $N=$ Controle Normotenso, $H=$ Controle Hipertenso, $C=$ Hipertenso tratado com Captopril.

\section{DISCUSSÃO}

O modelo utilizado para indução da hipertensão arterial nos animais deste estudo foi originalmente proposto por Goldblatt, em 1934, sugerindo que a isquemia renal levaria à hipertensão ${ }^{3}$. Este modelo vem sendo largamente testado e aperfeiçoado sendo, hoje, o modelo mais comumente utilizado para indução da hipertensão arterial nos estudos experimentais, em ratos. Vários autores utilizaram modelos experimentais de estenose unilateral de artéria renal e observaram que
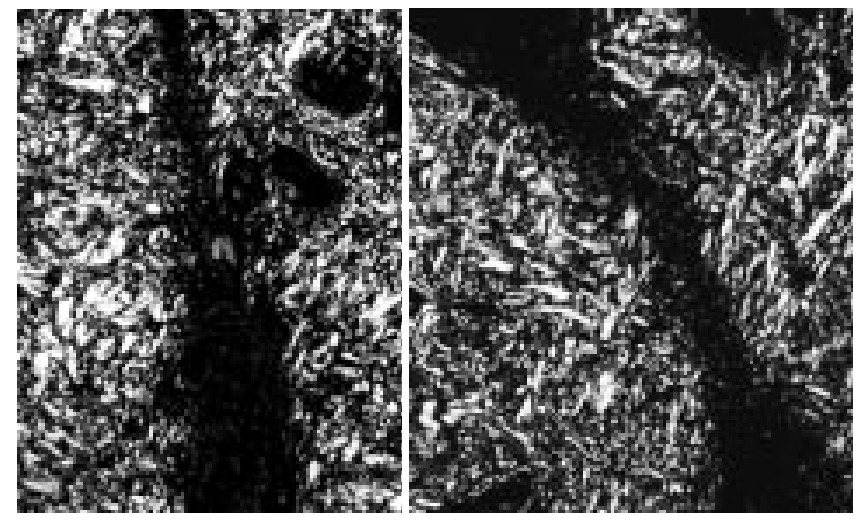

Figura 3 - Fotomicrografias de cortes histológicos demonstrando a presença do colágeno. Em A tem-se aspectos do grupo controle normotenso e em B, do grupo hipertenso tratado com captopril (Sirius Red $-100 \mathrm{X})$. 
este método é eficaz na indução de hipertensão arterial sistêmica de origem reno-vascular ${ }^{3,4,5}$.

Johns et al. estudaram diversos métodos de indução de hipertensão e de aferição da pressão arterial em ratos e observaram que as mensurações da pressão arterial tanto pelo método indireto utilizandoo "tail cuff" como pelo método direto feito com cateter intra-arterial são confiáveis e equivalentes entre $\mathrm{si}^{4}$

Não há registros de estudos sobre a interferência da hipertensão arterial e das drogas utilizadas para o seu tratamento no processo de cicatrização de pele.

Fawcett et al. estudaram a influência da hipertensão arterial na cicatrização de anastomoses colônicas e observaram que este fator aumenta a incidência de deiscência das anastomoses. Os autores sugeriram que este déficit de cicatrização ocorre por doença microvascular causada pela hipertensão, levando a fluxo sangüíneo reduzido no foco da anastomose ${ }^{6}$.

As alterações microvasculares da hipertensão arterial não se limitam apenas à microvasculatura colônica mas também ocorrem na tela subcutânea. As artérias do tecido subcutâneo de pacientes com hipertensão arterial essencial apresentam redução do lúmen arterial às custas do aumento da espessura da camada média da parede do vaso. Não há, porém, um aumento individual do tamanho e do número de células (miócitos $)^{7}$. Ficou demonstrado que a composição e a mecânica das artérias de resistência da tela subcutânea de pacientes com hipertensão arterial essencial mostram firmeza dos componentes da parede diminuída nas artérias de resistência. Parece que isto se deve às mudanças da arquitetura da matriz extra-celular ou da adesão célula-matriz ${ }^{8}$. Entretanto foi demonstrada a existência de hipertrofia das células musculares microvasculares ${ }^{9}$.

A hipóxia tecidual é um fator sistêmico que interfere na cicatrização e que se constitui na privação dos nutrientes e do oxigênio necessários para as células, que são levados através do sistema microcirculatório. A hipóxia diminui a deposição de colágeno, inibe a atividade fagocitária e pode levar ao hipercrescimento de microorganismos pela diminuição da atividade leucocitária ${ }^{10}$.

Além de alterações microvasculares, a hipertensão reno-vascular promove alterações significativas nos níveis séricos de angiotensina II. Sabe-se que esta proteína tem ação vasoativa, promovendo vasoconstrição arteriolar renal e, pela ativação do sistema renina-angiotensina-aldosterona, desenvolvendo a hipertensão reno-vascular. Guan et al. estudaram o sistema renina-angiotensina na hipertensão reno-vascular promovida por estenose unilateral da artéria renal e observaram que há elevação dos níveis plasmáticos de angiotensina I e II ${ }^{11}$.

Considerando-se que a angiotensina II tem ação vasoativa renal questiona-se se as alterações microvasculares da hipertensão arterial são decorrentes da ação periférica desta proteína e se isto pode vir a interferir no processo de cicatrização.

Koibuchi et al. (1993) estudaram a interferência da angiotensina II na ativação do fator de crescimento de fibroblastos e no fator de transformação ß1 (TGF-ß1) em cul- turas celulares de músculo liso vascular de ratos e observaram que é o balanço entre os dois fatores que determina o controle do crescimento da musculatura dos vasos. O TGF-ß1 determina a resposta celular à angiotensina II e tem ação antiproliferativa, o que leva à hipertrofia das células musculares mediada pelo fator de crescimento de fibroblastos, na hipertensão arterial $^{12}$.

Fernandez et al.estudaram o comportamento do sistema vascular em olhos de coelhos aos estímulos da angiotensina II e concluíram que esta angiotensina é capaz de induzir neoangiogênese ${ }^{13}$.

Para esta pesquisa, utilizou-se o captopril no tratamento da hipertensão arterial do grupo experimento. Esta droga age inibindo a enzima conversora da angiotensina, bloqueando, assim, o sistema renina-angiotensina e, portanto, reduzindo os níveis séricos de angiotensina II.

Volpert et al. estudaram o comportamento do sistema vascular da córnea de coelhos aos estímulos do captopril e observaram que a droga é um potente inibidor da angiogênese. Os autores sugerem que isto se deva à ação inibidora da enzima conversora da angiotensina ${ }^{14}$ e Vogt et al. observaram que doses de $100 \mathrm{mg} / \mathrm{dia}$ de captopril têm o poder de inibir a angiogênese das lesões do Sarcoma de Kaposi ${ }^{15}$.

Qiu et al. consideraram que a angiotensina é fundamental para a cicatrização e testaram a hipótese de que o captopril, inibidor da produção desta proteína, prejudicaria a cicatrização de feridas cutâneas, em ratos. Os autores não encontraram diferenças na cicatrização da pele destes animais quando comparados com um grupo tratado com uma proteína que estimula a produção de angiotensina e concluíram que o uso oral de captopril não inibe a cicatrização ${ }^{16}$.

A neoangiogênese é uma das características histológicas mais importantes do tecido de granulação no processo de cicatrização de pele.

Neste estudo, supõe-se que os animais do grupo controle hipertenso deveriam ter níveis séricos de angiotensina II elevados e maiores que os que se esperaria encontrar nos grupos controle normotenso e experimento (tratados com captopril). Uma vez posto que a angiotensina II favorece a neoangiogênese e que este fator é importante no processo de cicatrização, seria de se esperar que a cicatrização do primeiro grupo fosse favorecida em relação aos outros dois. Sabendo, ainda, que o captopril inibe a neoangiogênese por bloquear a atividade da angiotensina, também seria de se esperar que o grupo tratado com captopril tivesse seu processo cicatricial prejudicado quando comparado com os outros dois grupos, ou ainda, tivesse cicatrização semelhante à do grupo controle normotenso já que, segundo Michel et al. a droga é efetiva na redução dos valores pressóricos de ratos hipertensos ${ }^{3}$. Entretanto, não se verificaram diferenças de qualidade da cicatrização entre os grupos hipertensos tratados e não tratados. Observou-se que a cicatrização foi melhor no grupo controle normotenso, traduzida pela maior densidade de colágeno no controle normotenso em todos os tempos e não havendo diferença significante entre hipertensos tratados e não tratados. Verificou-se baixa da densidade tanto do colágeno I quanto 
do colágeno III nos dois grupos hipertensos. As cicatrizes dos grupos hipertensos tratado e não tratado eram menos resistentes que as do grupo controle normotenso, nos tempos iniciais $(\mathrm{p}<0,05)$ e ao $14 .^{\circ}$ dia as resistências se igualaram. Não se verificou diferença significante entre o grupo tratado e o não tratado.

Isto permite dizer que estes dados concordam com os de Qiu et al. que afirmaram que o captopril não inibe a cicatrização cutânea, em $\operatorname{ratos}^{16}$. Contudo, mais estudos precisam ser realizados, e em outras espécies animais antes que se possa dizer que esta droga não modifica a cicatrização.

O captopril, em ratos, não é fator de modificação do processo cicatricial ficando as diferenças relacionadas à hipertensão.

\section{AGRADECIMENTOS}

Agradecemos à Prof ${ }^{\mathrm{a}}$ Dr $^{\mathrm{a}}$ Márcia Olandoski pela orientação estatística.

\begin{abstract}
Background: We evaluated the influence of captopril on the skin wound healing process of hypertensive rats. Methods: 111 rats were placed in 4 groups: normotensive control $(N=30)$; hypertensive control $(N=30)$, which received an oral daily dose of saline solution 0,9\%; group experiment $(N=31)$ was treated with $7.5 \mathrm{mg} / \mathrm{kg} /$ day of captopril; and an aferition group $(N=20)$ with 10 hypertensive and 10 normotensive animals in which arterial blood pressure was mesured in the aorta in the last day of the experiment. After 15 days of treatment, an skin incision of $4 \mathrm{~cm}$ was made in the animals. Samples of the dorsal wall scar were taken 4, 7 and 14 days after the last procedure. The wounds were excised and divided in 2 pieces. They were sent to tensiontrial and histological analysis. Results: The aferition group showed mean arterial blood pressure of $82.5 \pm 7.55 \mathrm{mmHg}$ in the normotensive animals and $150.5 \pm 10.66 \mathrm{mmHg}$ in the hypertensive ones. The resistance analysis showed that the scars of treated and untreated hypertensives were less resistant than those of normotensives rats in the initial days $(p<0.05)$ and that on the $14^{\text {th }}$ day the resistances became similar. There were no diferences among treated and untreated groups. Total collagen had higher density in normotensives rats throughout the study $(p<0.05)$ and there were no diferences among treated and untreated hypertensive rats. Epitelization, inflammatory response and granulation tissue formation were similar in all groups. Conclusion: Captopril, doesn't modify the wound healing process in rats, being the differences due to hypertension.
\end{abstract}

Key Words: Wound healing; Skin; Hypertension; Captopril; Collagen.

\section{REFERÊNCIAS}

1. Chobanian AV, Bakris GL, Black HR, et al. The seventh report of the joint national committee on prevention, detection, evaluation, and treatment of high blood pressure: the JNC 7 report. JAMA. 2003; 289(19):2560-72.

2. Opie LH. Angiotensin converting enzyme inhibitors: scientific basis for clinical use. 2 ed. New York: Authors' Publishing House. 1992; p. 177-82.

3. Michel JB, Dussaule JC, Choudat L, et al. Effects of antihypertensive treatment in one-clip, two kidney hypertension in rats. Kidney Int. 1986; 29(5):1011-20.

4. Johns C, Gravas I, Handy DE, et al. Models of experimental hypertension in mice. Hypertension. 1996; 28(6):1064-9.

5. Vasquez EC, Cabral AM, Moyses MR,et al. Modelos de experimentais do emprego de antagonistas do cálcio na hipertensão arterial. Arq Bras Cardiol. 1986; 46(4)245-50.

6. Fawcett A, Shembekar M, Church JS, et al. Smoking, hipertension, and colonic anastomotic healing; a combined clinical and histopathological study. Gut. 1996; 38(5):714-8.

7. Korsgarrd N, Aalkjaer C, Heagerty AM, et al. Histology, of subcutaneous small arteries from patients with essential hypertension. Hypertension. 1993; 22(4): 523-6.

8. Intengan HD, Deng LY, Li JS, et al. Mechanics and composition of human subcutaneous resistence arteries in essential hypertension. Hypertension. 1999; 33(1 Pt 2):569-74.

9. Rizzoni D, Porteri E, Guefi D, et al. Cellular Hypertrophy in Subcutaneous Small Arteries of Patients with Renovascular Hypertension. Hypertension. 2000; 35(4): 931-5.

10. Clark JJ. Wond repair and factors influencing healing. Crit Care Nurs Q. 2002; 25(1):1-12.
11. Guan S, Fox J, Mitchell KD, et al. Angiotensin and angiotensin converting enzyme tissue levels in two-kidney, one-clip hipertensive rats. Hypertension. 1992; 20(6):763-7.

12. Koibuchi Y, Lee WS, Gibbons GH, et al. Role of Transforming Growth Factor- $\beta 1$ in the Cellular Growth Response to Angiotensin II. Hypertension. 1993; 21(6 Pt 2): 1046-50.

13. Fernandez LA, Twickler J, Mead A. Neovascularization produced by angiotensin II. J Lab Clin Med. 1985; 105(2):141-5.

14. Volpert OV, Ward WF, Lingen MW, et al.. Captopril inhibits angiogenesis and slows the growth of experimental tumors in rats. J Clin Invest. 1996; 98(3):671-9.

15. Vogt B, Frey FJ. Inhibition of angiogenesis in Kaposi ‘s sarcoma by captopril. Lancet . 1997; 349(9059):1148.

16. Qiu JG, Factor S, Chang TH, et al. Wound healing: captopril, an angiogenesis ihibitor, and Staphylococus aureus peptideoglycan. J Surg Res. 2000; 92(2):177-8

Como citar este artigo:

Biondo-Simões ML, Alcântara EM, Dallagnol JC, Yoshizumi KO, Torres LF, Borsato KS. Cicatrização de feridas: estudo comparativo em ratos hipertensos não tratados e tratados com inibidor da enzima conversora da angiotensina. Rev Col Bras Cir. [periódico na internet] 2006 Mar-Abr; 33(2). Disponível em URL: http://scielo.br/rcbc

Endereço para correspondência

Maria de Lourdes Pessole Biondo-Simões

Rua Ari José Valle, 1987 - Santa Felicidade

82030-000- Curitiba - Paraná

Telefone 0XX41. 3297.43.59

E-mail:biondo@avalon.sul.com.br 